
\title{
28 Research Square \\ Harnessing the Protein Corona towards Surface Design for Nanoparticle Tumor Delivery
}

\section{Shijiao Ma}

China Agricultural University

Junjie Xu

China Agricultural University

Jinxin He

Shanxi Agricultural University

Shuli Li

China Agricultural University

Haolan Zheng

China Agricultural University

\section{Bo Pang}

China Agricultural University

\section{Ying Wen}

China Agricultural University

\section{Qiaojun Fang}

National Center for Nanoscience and Technology

\section{Weiquan Liu}

China Agricultural University

Jiesheng Tian ( $\nabla$ tianhome@cau.edu.cn)

China Agricultural University

\section{Research}

Keywords: affibody, protein corona, anti-cancer therapy, targeted drug delivery, magnetosomes (BMPs), breast cancer cells, HER2, Trastuzumab

Posted Date: July 14th, 2021

DOI: https://doi.org/10.21203/rs.3.rs-683547/v1

License: (c) (i) This work is licensed under a Creative Commons Attribution 4.0 International License. Read Full License 


\section{Abstract \\ Background}

Nanoparticles (NPs) decorated with functional ligands (e.g., antibodies) are promising candidates for cancer diagnosis and treatment. However, many studies have shown that chemically coupled targeting moieties on NPs lose their targeting capability in biological milieu because they are shielded or covered by a "protein corona". It is highly desirable to increase the proportion of targeting moieties on the surface of NP-corona complex for purposes of cancer therapy.

\section{Results}

We designed and implemented an alternative engineering strategy for targeted drug delivery by linking a recombinant affibody (RA) scaffold to magnetosomes (BMPs). Pre-adsorption of BMP-RA with anti-HER2 humanized mAb trastuzumab (TZ) resulted in formation of BMP-RA-TZ complex. Linking of TZ to BMP via glutaraldehyde (GA) produced BMP-GA-TZ, which was used as control. Both engineered BMPs contained large amounts of TZ ( $0.2 \mathrm{mg}$ per mg BMP); however, TZs in BMP-RA-TZ were oriented in a consistent manner with HER2 binding site (Fab) toward the outside, whereas TZs in BMP-GA-TZ were oriented randomly. Degree of HER2 binding to BMP-RA-TZ was > 3x higher than that to BMP-GA-TZ. Incubation of BMP-RA-TZ and BMP-GA-TZ with normal human plasma or IgG-supplemented plasma resulted in alteration of surface proteins because of corona formation. GA-TZ-containing BMPs, in comparison with RA-TZ-containing BMPs, had larger hydrated radii and more surface proteins. The TZcontaining BMPs all could be targeted to and internalized in HER2-overexpressing breast cancer cell line SK-BR-3; however, their targeting efficiencies varied considerably: $50-75 \%$ for RA-TZ-containing BMPs, but only $9-19 \%$ for GA-TZ-containing BMPs. To simulate human body tumor environment, we incubated BMPs, plasma (100\%), and cancer cells together. In this milieu, BMP-RA-TZ uptake efficiency of SK-BR-3 was nearly $80 \%$ (slightly lower than for direct interaction with BMP-RA-TZ), whereas BMP-GA-TZ uptake efficiency was $<17 \%$.

\section{Conclusion}

Components of NP protein corona can be modified by linking RA scaffold to NP surface. Application of RA scaffold promoted an oriented arrangement of targeting ligands and reduced the shielding effect of corona proteins. This engineering strategy avoids reduction by corona formation of targeting capability in drug (NP) delivery.

\section{Introduction}

Oncological diseases remain among the most common causes of human mortality worldwide, despite remarkable advances in medical science during recent decades ${ }^{[1]}$. Principal anti-cancer treatment 
strategies in current use, which include radiation therapy, chemotherapy, nucleic acid therapy, and immunotherapy, are frequently associated with severe side-effects related to systemic toxicity resulting from lack of tumor specificity ${ }^{[2-5]}$. One approach for overcoming this problem is engineering of nanoparticle (NP) surfaces for targeting of tumors through addition of various synthetic or biological ligands, e.g., small molecules, peptides/ proteins, and antibodies (Abs) ${ }^{[6]}$. Targeting with NP-based platforms can enhance efficacy of drug delivery to specific tumor cells with relatively few adverse effects, thus improving diagnosis and therapy. This has been an exciting and fast-progressing research area in recent decades ${ }^{[6]}$.

One problematic issue is that host biological conditioning causes immediate adsorption to the NP surface upon blood or tissue contact. A variety of unpredictable and uncontrollable biomolecules, notably proteins, rapidly coat the NP surface and form a protein coating, termed "corona" ${ }^{[7]}$. The presence of a protein corona often results in reduction or elimination of NP targeting capability by shielding or completely covering the relevant functional groups ${ }^{[8]}$. Numerous strategies have been tested using surface barrier layers (polymer, protein, or biomimetic coatings) to block adhesion of corona proteins and prolong blood circulation time of NPs ${ }^{[9]}$. However, some of these strategies were found to inhibit internalization of NPs by cancer cells, resulting in limited therapeutic effectiveness (e.g., the "PEG dilemma") ${ }^{[10]}$. Some groups have introduced various types of cleavable bonds and mechanisms in engineered NPs, to prolong exposure of targeting ligands until they reach the target sites ${ }^{[11]}$. However, the above strategies for reducing protein corona effects are generally complicated and not widely used because of the inherent difficulty of in vivo application to diverse types of cancer cells and their external stimuli. There is a clear need for development of simple, widely applicable NP systems with a more easily controlled corona for targeted drug delivery.

We describe here a novel, alternative strategy for targeted drug delivery, based on precoating of functionalized NPs with humanized anti-tumor Abs in an oriented arrangement. We used a type of biogenic NP termed magnetosomes. BMPs are specialized organelles characteristically found in magnetotactic bacteria, composed of membrane-enveloped magnetite $\left(\mathrm{Fe}_{3} \mathrm{O}_{4}\right)$ or greigite $\left(\mathrm{Fe}_{3} \mathrm{~S}_{4}\right)$ crystals, with diameters ranging from 30 to $120 \mathrm{~nm}^{[12]}$. The BMP surface has many active chemical groups, particularly primary amino groups ${ }^{[13]}$. We constructed an engineered BMP (termed BMP-RA) by covalent linkage to a recombinant affibody scaffold. Affibodies are small molecules (non-Ig proteins) that have high affinity toward specific protein targets. The affibody automatically bound Abs, and maintained their orientation on BMP surface ${ }^{[14]}$. Incubation of BMP-RA with Ab resulted in orientation of antigen binding site (paratope) of $A b$ toward external BMP surface, thus promoting binding to antigen. Oriented host Abs presumably comprise the majority of protein corona of BMP-RA in biological milieu. Preadsorption of a humanized mAb (trastuzumab; TZ) onto BMP-RA resulted in attachment of fewer host compounds to Ab surface, and BMP-RA-TZ complexes retained their targeting capability even after formation of protein corona. 


\section{Materials And Methods \\ Cell lines and materials}

The magnetotactic bacterium Magnetospirillum gryphiswaldense MSR-1 is stocked in our laboratory. Cell lines SK-BR-3 (ATCC\#HTB-30) and MDA-MB-468 (ATCC口HTB-132) were kindly donated by National Center for Nanoscience and Technology. The anti-HER2 humanized mAb trastuzumab (TZ) was from Roche (Roche, Switzerland). McCoy's 5A medium, Leibovitz's L-15 medium, fetal bovine serum (FBS), and rabbit anti-HER2 Ab were from Thermo Fisher (Waltham, MA, USA). Human IgG was from Sigma-Aldrich (Germany). 4',6'-diamidino-2-phenylindole dihydrochloride (DAPI), 1,1-dioctadecyl-3,3,3,3tetramethylindocarbocyanine perchlorate (Dil), Prussian Blue Iron Stain Kit, and Enhanced BCA Protein Assay Kit were from Solarbio Science \&Technology (Beijing, China). Goat anti-rabbit IgG-HRP, rabbit antimouse IgG-HRP, rabbit anti-goat IgG-HRP, Peroxidase-AffiniPure F(ab')2 Fragment, and rabbit anti-goat IgG $(\mathrm{H}+\mathrm{L})$ were from Shanghai Universal Biotech Co.

\section{Human plasma}

Blood samples were taken from 10 healthy volunteer donors after obtaining informed consent for blood collection and subsequent analysis, and stored in 10-ml tubes containing $\mathrm{K}_{2}$ EDTA to prevent clotting. Samples were centrifuged $\left(1300 \times \mathrm{g}, 15 \mathrm{~min}, 4^{\circ} \mathrm{C}\right)$ to remove red and white cells, and supernatants were collected and stored at $-80^{\circ} \mathrm{C}$ for subsequent experiments.

\section{Magnetosome extraction}

M. gryphiswaldense MSR-1 were cultured in a 7.5-L fermentor (BioFlo 110; New Brunswick Scientific; CT, USA). Inoculum was cultured in sodium lactate medium as described in our 2008 report ${ }^{[15]}$. Cells was harvested at $3000 \mathrm{rpm}$ for $30 \mathrm{~min}$ at $4^{\circ} \mathrm{C}$. BMPs were extracted as described in our 2019 report ${ }^{[16]}$. In brief, harvested cells were resuspended in $10 \mathrm{mM}$ PBS (10 ml per $\mathrm{g}$ bacterial pellet; $\mathrm{pH}$ 7.4) and disrupted by ultrasonication (150 W, $30 \mathrm{~min}$ ) (model JY92-IIN; Scientz; Xiamen, China) on ice. BMPs were collected from solution by magnet at $4^{\circ} \mathrm{C}$ overnight, supernatant was discarded, and precipitate was resuspended in PBS. The above steps were repeated until protein in supernatant showed no decrease. BMPs were treated with $1 \mathrm{mg} / \mathrm{ml}$ proteinase $\mathrm{K}$ for $3 \mathrm{~h}$ at $56^{\circ} \mathrm{C}$, electroeluted as described in our 2011 report ${ }^{[17]}$, suspended in PBS at final concentration $1 \mathrm{mg} / \mathrm{ml}$, and sterilized by cobalt- 60 .

\section{Purification of recombinant protein A}

Nucleotides of protein A (Z domain) were optimized using a JAVA adaptation tool for soluble expression in E. coli BL21. Recombinant protein containing linker peptides and cysteine was synthesized at Nterminus, cloned into pET28(a+) plasmid with kanamycin-resistant gene, and transformed into E. coli $\mathrm{BL} 21$. The protein expressed by recombinant gene was termed RA. E. coli BL21 with plasmid was cultured in LB medium, expression was induced by addition over $8 \mathrm{~h}$ of isopropyl $\beta$-D-thiogalactopyranoside (IPTG) at final concentration $1 \mathrm{mM}$. Cells were collected by centrifugation $(10,000 \times g, 10 \mathrm{~min})$, resuspended in $10 \mathrm{mM}$ PBS (10 ml per g bacterial pellet; $\mathrm{pH}$ 7.4), disrupted by sonication at $30 \%$ 
amplitude (200 W, $30 \mathrm{~min})$ on ice, and centrifuged again (10,000 $\mathrm{g}$, $30 \mathrm{~min})$. RA protein present in supernatant was purified using HisTrap Fast Flow (FF) Crude Column as per manufacturer's instructions. Purified RA was freeze-dried and store at $-20^{\circ} \mathrm{C}$ for subsequent experiments.

\section{Identification of RA and its function}

Purified RA was added with protein loading buffer $(0.01(\mathrm{w} / \mathrm{v})$ bromophenol blue, $0.04 \mathrm{M}$ dithiothreitol (DTT), $10 \%$ glycerol, $2 \%\left(\mathrm{w} / \mathrm{v}\right.$ ) SDS, $62.5 \mathrm{mM}$ Tris-HCl, $\mathrm{pH} 6.8$ ), boiled for $10 \mathrm{~min}$ at $100^{\circ} \mathrm{C}$, and centrifuged. Supernatants were loaded onto $10 \%$ gels and run at $120 \mathrm{~V}$ for $1-2 \mathrm{~h}$ in a Mini-PROTEAN Tetra Electrophoresis System (Bio-Rad; USA). Gels were stained by Coomassie Brilliant Blue G250 for direct protein imaging, or transferred onto PVDF membrane in TransBlot SD Semi-Dry Electrophoretic Transfer Cell (Bio-Rad) for Western blotting. Membranes were blocked with $5 \%$ skim milk for $2 \mathrm{~h}$ at room temperature (RT), incubated with mouse anti-FLAG tag Ab (dilution 1:2000) for $8 \mathrm{~h}$, washed $3 \mathrm{x}$ with PBST (PBS containing $0.05 \%$ Tween-20), incubated with HRP-labeled goat anti-mouse Ab for $2 \mathrm{~h}$ at RT, and washed $3 x$ with PBST. Proteins on membranes were displayed using Immobilon Western chemiluminescent HRP substrate, and visualized using an imaging system (Fusion FX6; Vilber; France).

\section{Enzyme-linked immunosorbent assay (ELISA) analysis of functions of RA and RA-decorated BMPs}

The ability of RA to bind IgG was analyzed by ELISA. $2 \mu \mathrm{g}$ RA in $100 \mu$ l carbonate-bicarbonate buffer (pH 9.6) was incubated on 96 -well microtiter plate overnight at $4^{\circ} \mathrm{C}$. Plate was washed $3 x$ next day with PBST, added with $300 \mu \mathrm{l}$ of $1 \%$ BSA (diluted in PBST) for $1 \mathrm{~h}$ at RT to block nonspecific binding sites, washed $3 \mathrm{x}$ with PBST, added with same number of moles of rabbit anti-mouse IgG-HRP and rabbit anti-goat Peroxidase-AffiniPure F(ab')2 Fragment for $1 \mathrm{~h}$ at RT, washed 3x with PBST, and added with $100 \mu \mathrm{l}$ of 3,3',5,5'-tetramethylbenzidine (TMB) solution. After $8 \mathrm{~min}$, reaction was stopped by adding $50 \mu \mathrm{l}$ of $2 \mathrm{M}$ $\mathrm{H}_{2} \mathrm{SO}_{4}$. Absorbance at wavelength $450 \mathrm{~nm}$ was read by microtiter plate reader (ELx800; BioTek, U.S.). Samples without RA input were used as negative controls.

For BMP-RA, microtiter plate was blocked with 1\% BSA overnight, washed 3x, added with $1 \mathrm{ng}$ BMP-RA, placed over magnet for 5 min to isolate complex, added with rabbit anti-mouse IgG-HRP (1:20000 dilution in PBS) for $1 \mathrm{~h}$ at RT, and subsequent steps were the same as for RA. BMP wild-type (BMP-WT) was used as negative control.

For binding of BMP to human epidermal growth factor receptor-2 (HER2), initial processing of microtiter plate was the same as for BMP-RA. Then, plate was added with BMPs, washed, added for $1 \mathrm{~h}$ with HER2 at RT, washed $3 x$ with PBST, added with $100 \mu$ rabbit anti-HER2 Ab, incubated for $1 \mathrm{~h}$ with BMPs at RT, washed $3 x$, added with goat anti-rabbit IgG-HRP for $1 \mathrm{~h}$, and subsequent steps were the same as for BMPRA.

\section{Preparation of functional BMPs}


TZ functionalized BMPs were prepared using $N$-succinimidyl 3-(2-pyridyldithio) propionate (SPDP) and glutaraldehyde as cross-linkers, and termed BMP-RA-TZ and BMP-GA-TZ respectively. For BMP-RA preparation, 1 mg BMP was resuspended in HEPES (10 mM, pH 8.0) containing 1 mM SPDP, solution was ultrasonicated for $1 \mathrm{~min}$ followed by 1-min intervals (this step was repeated 30x), and mixture was washed $3 x$ with HEPES. RA $(1 \mathrm{mg} / \mathrm{ml})$ was suspended in $10 \mathrm{mM}$ HEPES, ultrasonicated for $1 \mathrm{~min}$ followed by 1 -min intervals (repeated $30 x$ ), supernatant was removed and washed $3 x$ with HEPES under magnet, and resuspended in HEPES. The BMP-complex was termed BMP-RA. SDS-PAGE was used to confirm RA linkage on magnetosome, $0.2 \mathrm{mg}$ BMP-RA was loaded, and subsequent steps were the same as for RA. For BMP-RA-TZ preparation, TZ solution $(500 \mu \mathrm{g} / \mathrm{ml})$ was suspended with modified BMPs and incubated for $2 \mathrm{~h}$ at $37^{\circ} \mathrm{C}$ with rotation (200 rpm). BMP-RA-TZ was washed $6 \mathrm{x}$ with HEPES and stored at $4^{\circ} \mathrm{C}$. BMP-GA-TZ was prepared as described in our 2019 report ${ }^{[18]}$. For confocal laser scanning microscopy (CLSM) and flow cytometry (fluorescence activated cell sorting; FACS), 1 mg BMP was FITClabeled by mixing with $300 \mu \mathrm{l}$ of $1 \mathrm{mg} / \mathrm{ml}$ FITC and incubated overnight at $4^{\circ} \mathrm{C}$ with shaking.

\section{Plasma-BMP interaction}

BMP samples were incubated with human plasma or IgG-enriched human plasma for $2 \mathrm{~h}$ at $37^{\circ} \mathrm{C}$ with shaking (200 rpm), and washed 6x with HEPES. For IgG-enriched human plasma experiments, $1 \mathrm{ml}$ human normal plasma was added with $6 \mathrm{mg} \mathrm{IgG}$. Following the last washing step, BMPs were resuspended with $1 \mathrm{ml} \mathrm{HEPES}$ in a 1.5-ml Eppendorf tube.

\section{Characterization of BMPs}

Transmission electron microscopy (TEM): BMP sample $(0.2 \mathrm{mg})$ was suspended in $1.5-\mathrm{ml}$ Eppendorf tube with $1 \mathrm{ml}$ double distilled water $\left(\mathrm{ddH}_{2} \mathrm{O}\right)$, ultrasonicated for $1 \mathrm{~min}$, and BMPs were collected under magnet and resuspended in $1 \mathrm{ml} \mathrm{ddH_{2 }}$ O. These steps were repeated $3 x .10 \mu \mathrm{L}$ of this suspension was dropped onto copper grid and air dried for $10 \mathrm{~min}$. Samples were observed by TEM (model JEM-1230; JEOL; Tokyo, Japan).

Zeta-potential and DLS measurement: BMP sample was resuspended in $\mathrm{ddH}_{2} \mathrm{O}$ at concentration 0.01 $\mathrm{mg} / \mathrm{ml}$ and ultrasonicated for $10 \mathrm{~min}$. Samples were combined in sample pool and measured using Zetasizer Nano ZS particle size analyzer (Malvern Instruments; Worcestershire, UK) at $25^{\circ} \mathrm{C}$.

\section{SDS-PAGE analysis}

Immediately following the last washing step, defined amount of BMP-corona complex $(0.2 \mathrm{mg})$ was resuspended in protein loading buffer, boiled for $10 \mathrm{~min}$ at $100^{\circ} \mathrm{C}$, BMPs were removed by centrifugation $(12,000 \times \mathrm{g}, 20 \mathrm{~min})$, and equal sample volume was loaded on $10 \% \mathrm{gel}$. SDS-PAGE was performed at $80 \mathrm{~V}$ for $30 \mathrm{~min}$, switched to $120 \mathrm{~V}$ for $\sim 90 \mathrm{~min}$ until bromophenol blue neared the gel end, and gel was stained with protein silver stain kit.

\section{Quantitative analysis of TZ on BMPs}


TZ supernatant before and after coupling to BMP was stored in advance, and analyzed using Enhanced BCA Protein Assay Kit as per manufacturer's protocol. Standard curve was constructed based on eight BSA concentration points $(0,0.025,0.05,0.1,0.2,0.3,0.4,0.5 \mathrm{mg} / \mathrm{ml}) .20 \mu \mathrm{l}$ samples or BSA (control) were placed on 96-well plates, added with $200 \mu \mathrm{lBCA}$ working solution, mixed thoroughly, incubated for $30 \mathrm{~min}$ at $37^{\circ} \mathrm{C}$, and read by microplate spectrophotometer (Power Wave XS2; BioTek) at $562 \mathrm{~nm}$. Sample concentrations were calculated based on the standard curve. Amount of TZ coupled to BMP was calculated as TZ amount before coupling -TZ amount TZ after coupling.

\section{Protein identification and classification}

BMP-corona complexes were obtained by the above methods, and proteins from SDS-PAGE $10 \%$ gels were excised and in-gel trypsin-digested by the method of Shevchenko et al. ${ }^{[19]}$. In brief, $100 \mu \mathrm{g}$ protein was dissolved in $50 \mathrm{mM}$ ammonium bicarbonate (ABC) solution, reduced with DTT for $45 \mathrm{~min}$ at $56^{\circ} \mathrm{C}$, alkylated with iodoacetamide (IAM) for $30 \mathrm{~min}$ at RT in the dark, and the solution was transferred to $10 \mathrm{~K}$ ultrafiltration tube (Vivacon 500; Sartorius, USA) and centrifuged (14000 x g, 20 min). Proteins were washed with $50 \mathrm{mM} \mathrm{ABC}$, added with $2 \mu \mathrm{g}$ trypsin in $50 \mu \mathrm{ABC}$, and incubated overnight at $37^{\circ} \mathrm{C}$. The ultrafiltration tube was centrifuged ( $14000 \times$ g, $20 \mathrm{~min}$ ), digested peptides were transferred to a collection tube, and $A B C$ was added to ultrafiltration tube to wash digested peptides into collection tube. Collected solution was diluted with $0.1 \%$ formic acid (FA) for nano-LC-MS analysis. NanoAcquity nano-HPLC (Waters Corp.; Milford, MA, USA) was used for nano-LC separation. Trap column was Thermo Acclaim PepMap $100(75 \mu \mathrm{m} \cdot 2 \mathrm{~mm}, \mathrm{C} 18,3 \mu \mathrm{m})$, and custom-made analytical column was fused silica capillary (I.D. $100 \mu \mathrm{m}$; Polymicro Technologies) filled with $20 \mathrm{~cm}$ stationary phase (Aqua 3- $\mu \mathrm{m}$ C18 125A; Phenomenex). Gradient elution program: mobile phase increased linearly from $1 \%$ B to $35 \%$ B in 65 min. Mobile phase $A$ was $0.1 \%$ FA in water; mobile phase $B$ was $0.1 \%$ FA in acetonitrile. Protein false discovery rate was set at $4 \%$, maximum protein mass was set at $600 \mathrm{kD}$, and generated peptide masses were compared to a reviewed human protein sequence database downloaded from UniProt (www.uniprot.org). Identification of a peptide required at least two assigned fragments; identification of a protein required at least two assigned peptides and five assigned fragments.

\section{Targeted cell uptake evaluation of BMPs by flow cytometry and CLSM}

To evaluate uptake of BMP-RA-TZ or BMP-GA-TZ by SK-BR-3 following incubation with human plasma or IgG-enriched human plasma, $\sim 1 \cdot 10^{5}$ cells in $1 \mathrm{ml}$ McCoy's $5 \mathrm{~A}$ medium with $10 \%$ FBS were seeded in a 12well plate, cultured for $24 \mathrm{~h}$ under standard conditions, and then incubated with various samples (BMPRA-TZ, BMP-RA-TZ-plasma, BMP-RA-TZ-IgG plasma, BMP-GA-TZ, BMP-GA-TZ-plasma, BMP-GA-TZ-IgG plasma) of BMPs $(10 \mu \mathrm{g} / \mathrm{ml})$ in McCoy's $5 \mathrm{~A}(0 \% \mathrm{FBS})$ for $2 \mathrm{~h}$. For analysis of BMP uptake at various plasma concentrations, $1 \cdot 10^{5}$ cells were placed in McCoy's $5 \mathrm{~A}$ with $0 \%, 10 \%$, or $100 \%$ human plasma, incubated with BMPs at indicated concentrations for $2 \mathrm{~h}$, and washed $2 \mathrm{x}$ with $10 \mathrm{mM}$ PBS. For flow cytometric measurements, cells were treated with $0.5 \%$ trypsin-EDTA, collected by centrifugation, washed $3 x$ with PBS $(10 \mathrm{mM})$, resuspended in $400 \mu \mathrm{PBS}$, and FITC fluorescence was measured with a flow 
cytometer (FACS Calibur; BD Biosciences; San Jose, CA, USA). Cells without BMP were used as negative control, and percentage of FITC-positive cells was calculated relative to negative control value. For CLSM, cells were seeded in a 24-well plate with cell slide, incubated with BMPs, washed $2 x$ with $10 \mathrm{mM}$ PBS, stained with $200 \mu \mathrm{l}$ of $10 \mu \mathrm{M}$ 1,1'-dioctadecyl-3,3,3',3'-tetramethylindocarbocyanine perchlorate for 10 min at $37^{\circ} \mathrm{C}$, fixed with $4 \%$ polyoxymethylene (POM) for $10 \mathrm{~min}$ at RT, and stained with $200 \mu \mathrm{l}$ of $10 \mu \mathrm{g} / \mathrm{ml}$ 4,6-diamidino-2-phenylindole dihydrochloride for $10 \mathrm{~min}$ at $37^{\circ} \mathrm{C}$. Images showing cellular uptake and distribution of BMPs with FITC-fluorescence signals were obtained using an inverted CLSM instrument (model A1; Nikon, Japan). Uptake efficiency of MDA-MB-468 was evaluated by an analogous series of steps.

\section{HER2 expression in SK-BR-3 and MDA-MB-468 cells}

HER2 expression on SK-BR-3 and MDA-MB-468 cell surfaces was evaluated by CLSM and Western blotting. For CLSM, cells were seeded in 24-well plate with cell slide, incubated for $24 \mathrm{~h}$, fixed with $4 \%$ POM for 10 min at RT, incubated with normal goat serum (Solarbio) for $1 \mathrm{~h}$ at RT, incubated with rabbit anti-HER2 Ab (1:500 v/v, in 0.01 M PBS; Invitrogen; USA) at $4^{\circ} \mathrm{C}$ overnight, incubated with Alexa Fluor 488conjugated goat-anti-rabbit $\lg G(1: 1000 \mathrm{v} / \mathrm{v}$, in $0.01 \mathrm{M}$ PBS $)$ for $1 \mathrm{~h}$ in the dark, and stained with $10 \mu \mathrm{g} / \mathrm{ml}$ DAPI in the dark.

For Western blotting, cells were collected from incubated on 6-well plate, washed with PBS, and added with $250 \mu$ native lysis buffer (Solarbio) on ice for $10 \mathrm{~min}$. Supernatant was mixed with SDS-PAGE loading buffer, boiled at $100^{\circ} \mathrm{C}$ for $10 \mathrm{~min}$, centrifuged, loaded on $10 \%$ gel, transferred to PVDF membrane, and blocked with 5\% BSA. Membrane was incubated with rabbit anti-HER2 Ab (1:1000 v/v, in $0.01 \mathrm{M}$ PBS) overnight at $4^{\circ} \mathrm{C}$, washed $3 \mathrm{x}$ with TBST (PBS containing $0.05 \% \mathrm{v} / \mathrm{v}$ Tween 20 ), incubated with HRP-labeled goat anti-rabbit (1:20000 v/v, in $0.01 \mathrm{M} \mathrm{PBS})$ for $2 \mathrm{~h}$ at RT, and imaged using an imaging system (Fusion FX6; Vilber).

\section{Prussian blue staining}

Cells were seeded in 24-well plate with cell slide, cultured for $24 \mathrm{~h}$ under standard condition, incubated with $10 \mu \mathrm{g} / \mathrm{ml}$ BMPs for $2 \mathrm{~h}$, washed 3x, fixed with $4 \%$ POM for 10 min at RT, the iron was detected by Prussian blue staining; i.e., equal volumes of Perls stain A and B were mixed, added to fixed cells and tissue sections, and incubated for $30 \mathrm{~min}$ at $37^{\circ} \mathrm{C}$. Samples were then washed $3 \mathrm{x}$ with Millipore water, immersed in Nuclear Fast Red solution for 2 min, cell slide was washed 3x with Millipore water, and cellular uptake and distribution of BMPs were imaged using a Zeiss microscope (Axio Cam MR3; Germany).

\section{Statistics analysis}

Statistical analysis was performed using student t-test for two groups, and one-way ANOVA for multiple groups. All data are given as mean \pm standard deviation (S.D.). Difference are considered statistically significant as ${ }^{*} P<0.05 ;{ }^{* \star P}<0.01 ; * \star * P<0.001 ; * \star \star * P<0.0001$. 


\section{Results}

\section{Decoration of BMP with a scaffold fusion protein}

To maintain targeting capability, surface ligands on a functionalized NP must retain highly directional orientation. This requires that the BMP be decorated with a protein scaffold. We utilized $Z$ domain (an affibody) as scaffold. $Z$ domain is derived from B domain of Staphylococcal Protein A (SpA), which binds Abs at their fragment crystallizable $(\mathrm{Fc})$ region. Therefore, BMPs decorated with $Z$ domain (termed BMP$Z s$ ) also bind to Fc moiety of Abs. Abs have a basic structure that resembles the letter $Y$. The lower part of the $Y$ is the Fc moiety. The top part of the $Y$, the Ab fragment (Fab), has an antigen binding site, termed "paratope" ${ }^{[20]}$. When targeting Abs attach to BMP-Zs, their Fab moieties are oriented toward the outside of resulting complexes, to maintain targeting ability.

We constructed a gene fragment of a fusion protein, composed of FLAG tag, cysteine residue (TGC), and two repeat $Z$ domain units in tandem. Linker sequences in front of the $Z$ domain genes were GSGS and GSGSGS (Fig. 1a). The fragment was cloned into pET28(a+) plasmid and expressed in E. coli BL21(DE3). The resulting fusion protein had moieties of two $Z$ domain units belonging to a recombinant affibody, and is referred to hereafter as "RA". FLAG tag was used as a label in Western blotting of RA (Fig. 1b). ELISA analysis showed that purified RA could be adsorbed by intact rabbit anti-mouse-HRP Ab and by rabbit anti-goat IgG-HRP, but not by Fab moiety of rabbit IgG (Fig. 1c,d), indicating that the two Z domain units in RA retained activity. The cysteine residue had a free sulfhydryl group, which facilitated linking of RA to primary amino groups of BMPs using the bifunctional reagent SPDP. WT BMPs were isolated from $M$. gryphiswaldense MSR-1 and digested with proteinase K (Fig. 1e) to remove bacterial proteins on BMP surface and minimize experimental "noise" and other possible adverse effects. BMP-WT and RA were linked by SPDP (Fig. 1f); the resulting NP complex is hereafter referred to as "BMP-RA". BMP-RA actively adsorbed rabbit anti-mouse IgG-HRP, indicating that the $Z$ domain on BMP-RA retained Fc-binding activity (Fig. 1g).

\section{Construction of targeting BMPs with humanized $\mathrm{Ab}$}

To construct delivery NPs, targeting ligands were linked to BMP-RAs. TZ was used as functional ligand for targeting to HER2-overexpressing cancer cells. TZ is a humanized anti-HER2 mAb used for treatment of breast cancer and stomach cancer based on Ab-dependent cell-mediated cytotoxicity (ADCC). Humanized Abs have low immunogenicity, and avoid attachment of host molecules to target ligands [21]. Incubation of BMP-RAs with TZs led to immobilization of large amounts of TZs on the surface, and formation of BMP-RA-TZ complex. SDS-PAGE and protein assay kit were used to measure TZ amount in the complex. TZ directly linked to BMP-WT by glutaraldehyde (BMP-GA-TZ) was used as control. The engineered BMPs contained similar TZ amounts: $0.2085 \pm 0.04861 \mathrm{mg}$ per mg BMP-RA-TZ and $0.1827 \pm$ 0.0522 mg per mg BMP-GA-TZ (Fig. 2a,b). TZ also attached to BMP-WT by nonspecific adsorption (referred to as BMP-TZ), but the amount was much less than in BMP-RA-TZ and BMP-GA-TZ (Fig. S1). Despite similar linkage rates of BMP-RA-TZ and BMP-GA-TZ, orientation of TZs in these two 
functionalized NPs was theoretically quite different. TZs on BMP-RA-TZ were presumably highly ordered, with Fab moiety directed outward ${ }^{[22]}$, whereas TZs on BMP-GA-TZ were oriented in random fashion (Fig. 2c). This hypothesis was tested by ELISA analysis of antigen (HER2 protein) amounts on the two engineered BMPs following linkage to HER2. BMP-RA-TZ was found to adsorb $>3 x$ more HER2 than did BMP-GA-TZ (Fig. 2d).

\section{Effect of Ab precoating on BMP coronas}

In organisms, protein coronas may shield or cover active molecules at the NP surface, resulting in mistargeting or unintended scavenging by liver, kidney, or spleen ${ }^{[23]}$. We hypothesized that Ab binding scaffolds (RAs, in this case) can alter protein components and target ligand orientations in protein corona. We examined and compared protein coronas of our WT and engineered BMPs following incubation with human plasma. Because corona formation on BMPs following plasma incubation resulted in greatly altered surface layer composition (new biological identity) ${ }^{[24]}$, we referred to them by adding "-plasma" to the end of the original name; e.g., "BMP-RA-TZ-plasma" instead of "BMP-RA-TZ". IgG levels in blood may increase because of infections, autoimmune processes, inflammation, or malignancy [25]; we therefore performed an additional treatment by adding human IgG in normal plasma to simulate patients' physiological condition in vitro. For this situation, the phrase "-IgG plasma" was added to the end of the original name. IgGs are by far the most abundant Abs in plasma; our subsequent experiments therefore focused only on IgGs rather than all Abs.

Corona components of engineered BMPs were greatly altered. BMP-RA and BMP-GA, incubated with either normal plasma or IgG plasma, bound much more IgG in the corona than did BMP-WT (Fig. S2). IgG content of BMP-RA-TZ and BMP-GA-TZ coronas was similar to original TZ content (Fig. S3). Components of these BMP coronas were analyzed by liquid chromatography - mass spectrometry (LC-MS). For each type of corona, the 20 proteins with highest content are listed in Table S1. Protein species, amounts, and ratios in coronas were strongly affected by pre-adsorption of TZ and by type of TZ/ BMP linkage (Figs. S3, S4). IgG content in coronas was much higher for BMP-RA-TZ and BMP-GA-TZ than for BMP-WT. IgG content was slightly higher for BMP-RA-TZ-IgG plasma than for BMP-RA-TZ-plasma (Fig. S1). Protein content was higher for BMP-GA-TZ-plasma than for BMP-RA-TZ-plasma. Of 70 identified proteins, 42 species had higher content for BMP-GA-TZ-plasma than for BMP-RA-TZ-plasma. Average protein molecular weight (MW) was also higher for BMP-GA-TZ-plasma than for BMP-RA-TZ-plasma (Fig. 3b,c). Among the proteins with higher content in BMP-GA-TZ-plasma than in BMP-RA-TZ-plasma, $42.9 \%$ had $\mathrm{MW}>60 \mathrm{KD}$, and $57.1 \%$ had MW $\leq 60 \mathrm{KD}$. Among proteins with higher content in BMP-RA-TZ-plasma than in BMP-GA-TZ-plasma, 71.4\% had MW $\leq 60 \mathrm{KD}$. These findings indicate that for BMPs with oriented $\mathrm{TZ}$, fewer and smaller host compounds were incorporated into protein coronas.

TZ and human IgGs have similar affinities for RA; therefore, some human IgG molecules may competitively bind to BMP-RA-TZ, and replace or shield pre-adsorbed TZs. If a large proportion of TZs were repelled or shielded by human IgGs, BMP-RA-TZ-plasma and BMP-RA-TZ-IgG plasma would lose their targeting ability. To investigate these possibilities, we used double Ab sandwich ELISA to measure 
amounts of HER2 bound to BMP-RA-TZ before and after incubation with human plasma. In addition to BMP-GA-TZ, another control (termed BMP-RA-IgG) was constructed by incubating BMP-RA and commercial human IgG. BMP-RA-TZ bound higher amounts of HER2 following incubation with normal plasma than with IgG plasma, suggesting that IgGs could repel or shield some proportion of TZs. On the other hand, both BMP-RA-TZ-plasma and BMP-RA-TZ-IgG plasma bound much higher amounts of HER2 than did BMP-RA-IgG, BMP-GA-TZ-plasma and BMP-GA-TZ-IgG plasma, indicating that large amounts of TZ were still present on BMP-RA-TZ surface (Fig. 3d).

TZ pre-adsorption and corona formation affected hydrated radii and zeta potentials of the engineered BMPs. TZ pre-adsorption led to slight increase of hydrated radii and reduced absolute values of zeta potentials. Hydrated radii of GA-TZ-containing BMPs were larger than those of RA-TZ-containing BMPs (Fig. 3e,f). Effects of coronas on zeta potentials varied depending on type of BMP. Absolute values of BMP zeta potentials declined to the 10-30 mV range, with the exception of BMP-GA-TZ-plasma. A mixed dispersion system with zeta potential absolute value in this range is considered to have incipient stability ${ }^{20}$; thus, it appeared that TZ pre-adsorption and corona formation did not notably affect dispersity of BMPs.

\section{Targeting analysis of TZ functionalized BMPs}

Targeting capacities of the TZ functionalized BMPs were investigated by fluorescence microscopy and flow cytometric analysis. Because TZ is an anti-HER2 mAb, we used two human breast cancer cell lines for evaluation: SK-BR-3 (HER2-overexpressing) and MDA-MB-468 (HER2-negative) ${ }^{[26]}$. HER2 expression on cancer cell surface was determined by immunofluorescence assay. Anti-HER2 secondary Ab was labeled with Alexa Fluor 488 (bright green fluorescent dye), and cancer cell nuclei were stained by DAPI (blue). CLSM observation revealed surface expression of HER2 on SK-BR-3 but not MDA-MB-468 (Fig. S5a). Western blotting analysis gave similar results (Fig. S5b).

Interactions of BMPs with cancer cells were investigated by prestaining BMPs with FITC (green), and cell membranes with Dil (red). Interactions of SK-BR-3 with various BMPs were observed by CLSM. BMPs without RA (BMP-WT, BMP-GA-TZ, BMP-GA-TZ-plasma) were localized mainly outside (surrounding) cancer cells. In contrast, BMPs with RA (BMP-RA-TZ, BMP-RA-TZ-plasma, BMP-RA-TZ-IgG plasma) were internalized into cancer cells to some degree, in addition to surrounding the cells (Fig. 4a). These findings suggest that oriented TZ mediated entry of BMPs into HER2-positive cells. For purposes of flow cytometric analysis (below), cells with engineered BMPs on the surface and cells with some BMPs located internally were not easily distinguishable, and were referred to generally as FITC-positive cells.

Percentages of FITC-positive cells were calculated based on flow cytometry. Following incubation with BMP-RA-TZ, BMP-RA-TZ-plasma, and BMP-RA-TZ-IgG plasma, percentages of FITC-positive SK-BR-3 cells were respectively $72 \%, 61.9 \%$, and $55.2 \%$ (Fig. 4b,c,d). FITC-positive percentages were much lower for SKBR-3 incubated with other types of BMPs. BMP-WT, BMP-RA-IgG, BMP-WT-plasma, BMP-RA-IgG-plasma, BMP-WT-IgG plasma, and BMP-RA-IgG-IgG plasma showed essentially no interaction with SK-BR-3. BMPGA-TZ, BMP-GA-TZ-plasma, and BMP-GA-TZ-IgG plasma interacted to slight degrees with SK-BR-3, but 
FITC-positive percentages (respectively $19 \%, 9.85 \%$, and $14.8 \%$ ) were significantly lower than for RA-TZcontaining BMPs (Fig. 4b,c,d). These findings clearly indicate that targeting capacities of RA-TZcontaining BMPs were much stronger than those of GA-TZ-containing BMPs.

\section{Uptake of BMPs by cancer cells mediates TZ orientation}

In view of previous reports that cellular uptake of nanomaterials is greatly reduced by protein corona [27], we evaluated the possibility that TZ/ HER2 interaction mediates internalization of BMPs into breast cancer cells. Although CLSM observation results (preceding section) showed BMP uptake by some cancer cells, FITC is a dye for proteins; thus, the above results did not rule out the possibility of BMP disintegration when crossing cell membrane. Prussian blue staining, a method commonly used for detection of iron in biological samples, was applied for microscopic observation [28]. Under this procedure, BMPs were visualized as blue particles, and cancer cells were dyed red (Fig. S6). RA-TZ-containing BMPs were internalized into SK-BR-3, whereas other types of BMPs were not. These findings confirmed those shown in Fig. 4a, and indicate that only oriented TZs can efficiently internalize BMPs into cancer cells.

To simulate human body tumor environment, we incubated BMPs, plasma (100\%), and cancer cells together, with staining procedures as described in the preceding section. Treatments with $0 \%$ and $10 \%$ plasma were performed to evaluate effects of plasma concentration on BMP uptake. CLSM observation showed that BMP-RA-TZ was internalized into SK-BR-3 at each of these three plasma levels, whereas BMP-WT was not (Fig. 5a). Flow cytometric analysis revealed FITC-positive percentages in $0 \%, 10 \%$, and $100 \%$ plasma of (respectively) $86.5 \%, 84.6 \%$ and $79.4 \%$ for BMP-RA-TZ, and $16.4 \%, 11.7 \%$ and $2.61 \%$ for BMP-GA-TZ (Fig. 5b). These findings indicate that oriented TZ was required for efficient internalization of BMPs into cancer cells, and that plasma concentration had negligible affect on BMP uptake.

The hypothesis that TZ/ HER2 interaction mediates BMP uptake by cancer cells was tested using HER2negative MDA-MB-468 as negative control. MDA-MB-468 showed no FITC fluorescence when incubated with WT or engineered BMPs (Fig. S7). Flow cytometric analysis in experiments with all BMPs showed that $<5 \%$ of MDA-MB-468 cells were FITC-positive. These findings indicate that MDA-MB-468 were incapable of BMP update because of lack of HER2, and confirm the essential role of oriented TZ in recognition of HER2, and in BMP uptake by cancer cells.

\section{Discussion}

Until the 1980s, the primary category of therapeutic agents was small-molecule (<900 Da) drugs (e.g., chemotherapeutics, antibiotics, steroids). However, only 10\% of the human genome can be targeted by small-molecule drugs ${ }^{[29]}$. New therapeutic functions are possible for "new generations" of therapeutic agents developed in recent decades, which include proteins, peptides, mAbs, nucleic acids, and living cells [30]. Antibodies, in particular, have great potential as drugs because of their high target specificity ${ }^{[31]}$. As of 2020 , antibodies were the subjects of $>500$ ongoing clinical trials and $>90$ clinical approvals by the U.S. Food and Drug Administration (FDA) ${ }^{[32,33]}$. However, the very short half-life of therapeutic Abs 
means that application of Ab-based therapy requires multiple administrations and high cost. Ab-based therapy also involves low degree of tissue penetration. Use of NPs as Ab delivery systems allows reduction of $A b$ dosing and increased Ab stability ${ }^{[34]}$. Magnetic NPs (MNPs) are of particular interest as a basis for magnetic drug delivery, because they can be easily conjugated with Abs and delivered to target organs ${ }^{[35]}$. BMPs, as a MNP candidate for drug delivery, have the advantages of single magnetic domain, high crystallinity, strong biocompatibility, and easy surface modification ${ }^{[36]}$. The external boundary of BMPs is a biomembrane, and the large amounts of functional groups on surface proteins and lipids [13] facilitates chemical and genetic engineering. In the present study, BMPs adsorbed $\sim 200 \mu \mathrm{g}$ lgGs per mg, in contrast to $1.5 \mu \mathrm{g}$ proteins per mg of iron oxide NPs in a previous study [37].

A NP-biomolecule (NP-corona) complex is formed following administration of NP into biological fluids, and constitutes the "true identity" of NP in the human body. Ungoverned adsorption of biomolecules onto NPs has generally negative effects, and inhibits accessibility of NP targeting ligands ${ }^{[38]}$. Mechanisms of corona formation remain poorly understood; however, numerous studies have characterized and identified corona proteins from patient samples ${ }^{[39]}$. Manipulation and utilization of coronas has potential therapeutic benefits in terms of drug delivery and targeted cancer treatment, and research in progress is focused on prolonging blood circulation time and enhancing targeting efficiency of NPs ${ }^{[11]}$.

Coronas of BMPs have not been analyzed until last year ${ }^{[37]}$. We developed and describe here a simple alternative design strategy for directed formation of NP protein corona. Protein scaffolds on BMP surface favor recruitment of desired proteins (IgG, in this case) in plasma during corona formation. Full human or humanized $\mathrm{Abs}$ ( $\mathrm{TZ}$, in this case) can be incorporated (by pre-adsorption) into corona as targeting ligands. Spatial orientation of these Abs can be controlled to enhance their interaction capability with target molecules, and uptake by target cells. Our findings indicate that most pre-adsorbed host molecules on protein scaffolds are retained in the corona and maintain their desired functions. In this new design strategy, corona formation is not viewed as a passive obstruction to NP delivery, but rather as a contributor to "NP identity"; i.e., NP targeting capacity is mediated by specific components (IgG, in this case) in the corona.

\section{Conclusion}

A new design strategy for nanoparticle (NP) construction was developed and implemented. The strategy took protein corona into account, with NP-corona complex considered as final identity of functionalized NP. A Z domain-based recombinant affibody (RA) scaffold was linked to BMPs, which were then preadsorbed on anti-HER2 humanized mAb (TZ). Following incubation with human plasma, most of the preadsorbed TZ molecules were incorporated into NP-corona complex, and maintained their targeting function. RA kept TZ molecules in a defined spatial orientation that favored binding to the target antigen (HER2), as demonstrated by the much higher efficiency in targeting and cellular uptake of BMP-RA-TZplasma and BMP-RA-TZ-IgG plasma complexes, relative to counterpart complexes in which BMP and TZ were directly linked to chemical bifunctional reagent. 


\section{Abbreviations}

Nanoparticle: NP Magnetosome: BMPs trastuzumab: TZ

Recombinant affibody: RA Glutaraldehyde: GA

$N$-succinimidyl 3-(2-pyridyldithio) propionate: SPDP

Fetal bovine serum: FBS

Enzyme-linked immunosorbent assay: ELISA Confocal laser scanning microscopy: CLSM

\section{Declarations}

\section{Authors' contributions}

SjM, JsT and WqL conceived and designed the experiments. SjM, JxH, JjX, SIL, HIZ and BP performed the experiments. SjM, JsT, and WqL analyzed the data., SjM, JsT drafted and wrote the manuscript. All authors read and approved the final manuscript.

\section{Competing interests}

The authors declare that they have no competing interests.

\section{Consent for publication}

All authors have provided consent for the manuscript to be published

\section{Funding}

This study was supported by Key Project of Inter-Governmental International Scientific and Technological Innovation Cooperation (2019YFE0115800), National Natural Science Foundation of China (No. $31570037,21577170)$, and the Project for Extramural Scientists of State Key Laboratory of Agrobiotechnology (2020SKLAB6-6), Beijing Municipal Science and Technology Project (Z201100007920010).

\section{Acknowledgements}

We thank Beijing Guokerongzhi Bio-Technology Co., Ltd for the help in magnetosome preparation. The authors are also grateful to Dr. S. Anderson for English editing of the manuscript.

\section{References}


1. Feigin VL, Nichols E, Alam T, Bannick MS, Beghi E, Blake N, Culpepper WJ, Dorsey ER, Elbaz A, Ellenbogen RG, et al. Global, regional, and national burden of neurological disorders, 1990-2016: a systematic analysis for the Global Burden of Disease Study 2016. Lancet Neurol. 2019;18(5):45980.

2. Alterio D, Marvaso G, Ferrari A, Volpe S, Orecchia R, Jereczek-Fossa BA. Modern radiotherapy for head and neck cancer. Semin Oncol. 2019;46:233-45.

3. Schirrmacher V. From chemotherapy to biological therapy: A review of novel concepts to reduce the side effects of systemic cancer treatment (Review). Int J Oncol. 2019;54:407-19.

4. Scotte F, Ratta R, Beuzeboc P. Side effects of immunotherapy: a constant challenge for oncologists. Curr Opin Oncol. 2019;31:280-5.

5. Stewart MP, Sharei A, Ding X, Sahay G, Langer R, Jensen KF. In vitro and ex vivo strategies for intracellular delivery. Nature. 2016;538:183-92.

6. Salvioni L, Rizzuto MA, Bertolini JA, Pandolfi L, Colombo M, Prosperi D. Thirty years of cancer nanomedicine: success, frustration, and hope. Cancers. 2019;11:1855.

7. Rampado R, Crotti S, Caliceti P, Pucciarelli S, Agostini M. Recent advances in understanding the protein corona of nanoparticles and in the formulation of "stealthy" nanomaterials. Front Bioeng Biotechnol. 2020;8:166.

8. Salvati A, Pitek AS, Monopoli MP, Prapainop K, Bombelli FB, Hristov DR, et al. Transferrinfunctionalized nanoparticles lose their targeting capabilities when a biomolecule corona adsorbs on the surface. Nat Nanotechnol. 2013;8:37-43.

9. Fam SY, Chee CF, Yong CY, Ho KL, Mariatulqabtiah AR, Tan WS. Stealth coating of nanoparticles in drug-delivery systems. Nanomaterials. 2020;10:787.

10. Zhu Y, Chen C, Cao Z, Shen S, Li L, Li D, et al. On-demand PEGylation and dePEGylation of PLA-based nanocarriers via amphiphilic mPEG-TK-Ce6 for nanoenabled cancer chemotherapy. Theranostics. 2019;9:8312-20.

11. Pinals RL, Chio L, Ledesma F, Landry MP. Engineering at the nano-bio interface: harnessing the protein corona towards nanoparticle design and function. Analyst. 2020;145:5090-112.

12. Uebe R, Schuler D. Magnetosome biogenesis in magnetotactic bacteria. Nat Rev Microbiol. 2016;14:621-37.

13. Han L, Li S, Yang Y, Zhao F, Huang J, Chang J. Comparison of magnetite nanocrystal formed by biomineralization and chemosynthesis. J Magn Magn Mater. 2007;313:236-42.

14. Gebauer M, Skerra A. Engineered Protein Scaffolds as Next-Generation Therapeutics. Annu Rev Pharmacol Toxicol. 2020;60:391-415.

15. Liu J, Ding Y, Jiang W, Tian J, Li Y, Li J. A mutation upstream of an ATPase gene significantly increases magnetosome production in Magnetospirillum gryphiswaldense. Appl Microbiol Biotechnol. 2008;81:551-58. 
16. Xu J, Liu L, He J, Ma S, Li S, Wang Z, et al. Engineered magnetosomes fused to functional molecule (protein A) provide a highly effective alternative to commercial immunomagnetic beads. $J$ Nanobiotechnology. 2019;17:37.

17. Guo F, Liu Y, Chen Y, Tang T, Jiang W, Li Y, Li J. A novel rapid and continuous procedure for largescale purification of magnetosomes from Magnetospirillum gryphiswaldense. Appl Microbiol Biotechnol. 2011;90:1277-83.

18. Geng Y, Wang J, Wang X, Liu J, Zhang Y, Niu W, Basit A, Liu W, Jiang W. Growth-inhibitory effects of anthracycline-loaded bacterial magnetosomes against hepatic cancer in vitro and in vivo. Nanomedicine. 2019;14(13):1663-80.

19. Shevchenko A, Wilm M, Vorm 0 , Mann M. Mass spectrometric sequencing of proteins silver-stained polyacrylamide gels. Anal Chem. 1996;68(5):850-8.

20. Kapingidza AB, Kowal K, Chruszcz M. Antigen-antibody complexes. Subcell Biochem. 2020;94:46597.

21. Waldmann H. Human Monoclonal Antibodies. The benefits of humanization. Methods Mol Biol. 2019;1904:1-10.

22. Tripathi K, Driskell JD. Quantifying bound and active antibodies conjugated to gold nanoparticles: A comprehensive and robust approach to evaluate immobilization chemistry. ACS Omega. 2018;3:8253-59.

23. Monopoli MP, Walczyk D, Campbell A, Elia G, Lynch I, Bombelli FB, Dawson KA. Physical-chemical aspects of protein corona: relevance to in vitro and in vivo biological impacts of nanoparticles. $\mathrm{J}$ Am Chem Soc. 2011;133:2525-34.

24. Nierenberg $D$, Khaled AR, Flores 0 . Formation of a protein corona influences the biological identity of nanomaterials. Rep Pract Oncol Radiother. 2018;23:300-8.

25. Notarangelo LD, Duse M, Ugazio AG. Immunodeficiency with hyper-IgM (HIM). Immunodefic Rev. 1992;3(2):101-21.

26. Mota AL, Evangelista AF, Macedo T, Oliveira R, Scapulatempo-Neto C, Vieira RA, Marques MMC. Molecular characterization of breast cancer cell lines by clinical immunohistochemical markers. Oncol Lett. 2017;13:4708-12.

27. Carter P, Presta L, Gorman CM, Ridgway JB, Henner D, Wong WL, Rowland AM, Kotts C, Carver ME, Shepard HM. Humanization of an anti-p185HER2 antibody for human cancer therapy. Proc. Natl. Acad. Sci. 1992;89(10):4285-9.

28. Hall AP, Davies W, Stamp K, Clamp I, Bigley A. Comparison of computerized image analysis with traditional semiquantitative scoring of Perls' Prussian Blue stained hepatic iron deposition. Toxicol Pathol. 2013;41:992-1000.

29. Milletti F. Cell-penetrating peptides: classes, origin, and current landscape. Drug Discov Today. 2012;17:850-60.

30. Vargason AM, Anselmo AC, Mitragotri S. The evolution of commercial drug delivery technologies. Nat Biomed Eng. 2021. doi:10.1038/s41551-021-00698-w. 
31. Marrocco I, Romaniello D, Yarden Y. Cancer immunotherapy: the dawn of antibody cocktails. Methods Mol Biol. 2019;1904:11-51.

32. Lu RM, Hwang YC, Liu IJ, Lee CC, Tsai HZ, Li HJ, Wu HC. Development of therapeutic antibodies for the treatment of diseases. J Biomed Sci. 2020;27:1.

33. Kaplon H, Reichert JM. Antibodies to watch in 2021. Mabs. 2021;13:1860476.

34. Sousa F, Castro P, Fonte P, Kennedy PJ, Neves-Petersen MT, Sarmento B. Nanoparticles for the delivery of therapeutic antibodies: Dogma or promising strategy? Expert Opin Drug Deliv. 2017;14:1163-76.

35. Peng XH, Qian X, Mao H, Wang AY, Chen ZG, Nie S, Shin DM. Targeted magnetic iron oxide nanoparticles for tumor imaging and therapy. Int J Nanomedicine. 2008;3(3):311-21.

36. Alphandery E. Applications of magnetotactic bacteria and magnetosome for cancer treatment: A review emphasizing on practical and mechanistic aspects. Drug Discov Today. 2020;25:1444-52.

37. Lai W, Li D, Wang Q, Nan X, Xiang Z, Ma Y, Liu Y, Chen J, Tian J, Fang Q. A Protein Corona Adsorbed to a Bacterial Magnetosome Affects Its Cellular Uptake. Int J Nanomedicine. 2020;15:1481-98.

38. Monopoli MP, Aberg C, Salvati A, Dawson KA. Biomolecular coronas provide the biological identity of nanosized materials. Nat Nanotechnol. 2012;7:779-86.

39. Kopac T. Protein corona, understanding the nanoparticle-protein interactions and future perspectives: A critical review. Int J Biol Macromol. 2021;169:290-301.

\section{Figures}



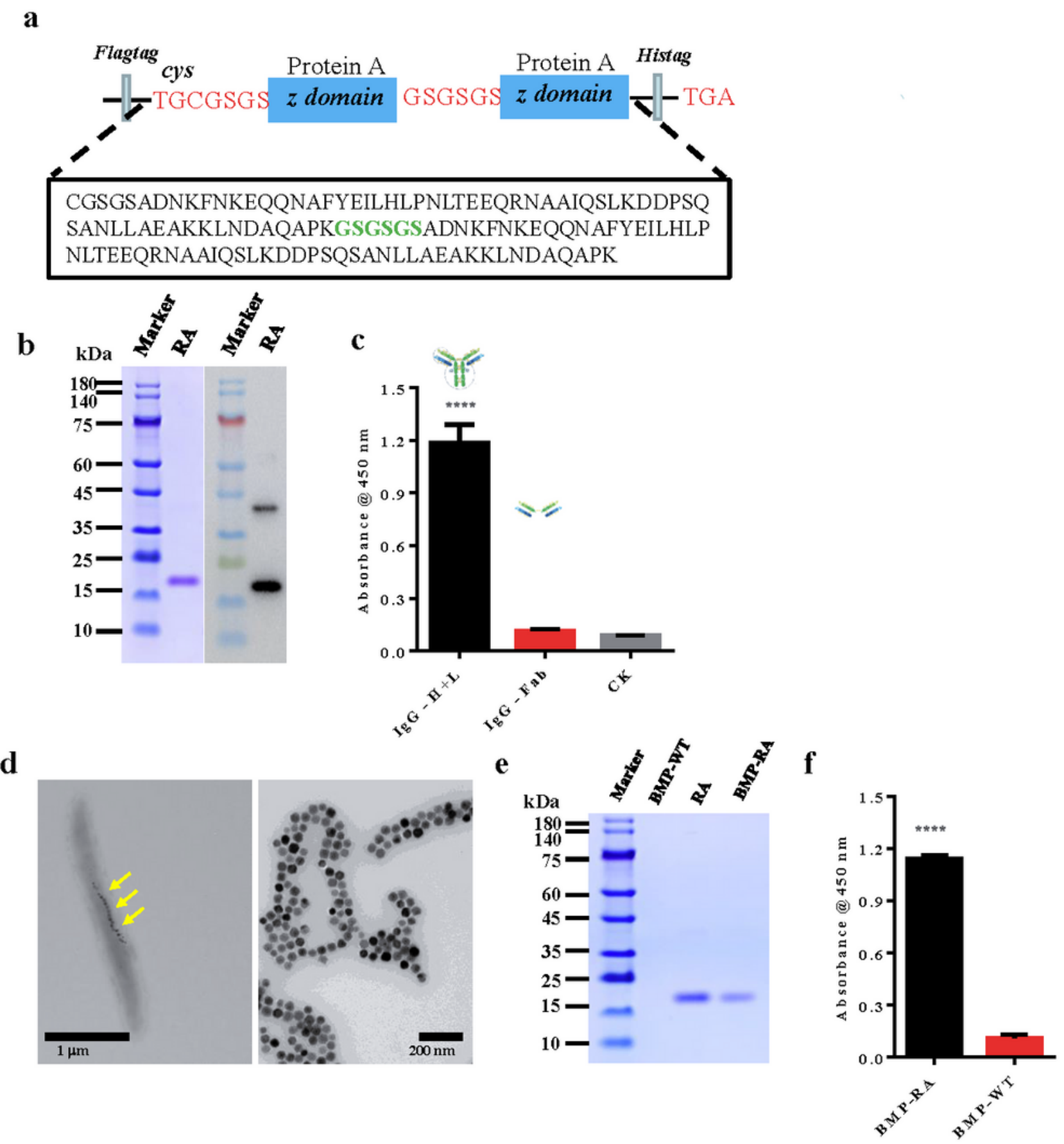

\section{Figure 1}

We constructed a gene fragment of a fusion protein, composed of FLAG tag, cysteine residue (TGC), and two repeat $Z$ domain units in tandem. Linker sequences in front of the $Z$ domain genes were GSGS and GSGSGS (Fig. 1a). The fragment was cloned into pET28(a+) plasmid and expressed in E. coli BL21(DE3). The resulting fusion protein had moieties of two $Z$ domain units belonging to a recombinant affibody, and is referred to hereafter as "RA". FLAG tag was used as a label in Western blotting of RA (Fig. 1b). ELISA 
analysis showed that purified RA could be adsorbed by intact rabbit anti-mouse-HRP Ab and by rabbit anti-goat IgG-HRP, but not by Fab moiety of rabbit IgG (Fig. 1c,d), indicating that the two $Z$ domain units in RA retained activity. The cysteine residue had a free sulfhydryl group, which facilitated linking of RA to primary amino groups of BMPs using the bifunctional reagent SPDP. WT BMPs were isolated from M. gryphiswaldense MSR-1 and digested with proteinase K (Fig. 1e) to remove bacterial proteins on BMP surface and minimize experimental "noise" and other possible adverse effects. BMP-WT and RA were linked by SPDP (Fig. 1f); the resulting NP complex is hereafter referred to as "BMP-RA". BMP-RA actively adsorbed rabbit anti-mouse IgG-HRP, indicating that the $Z$ domain on BMP-RA retained Fc-binding activity (Fig. 1g).

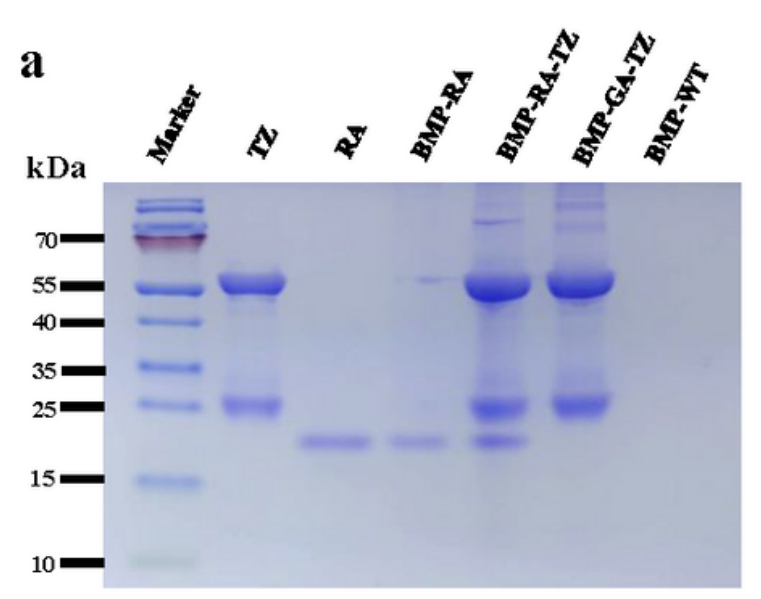

b
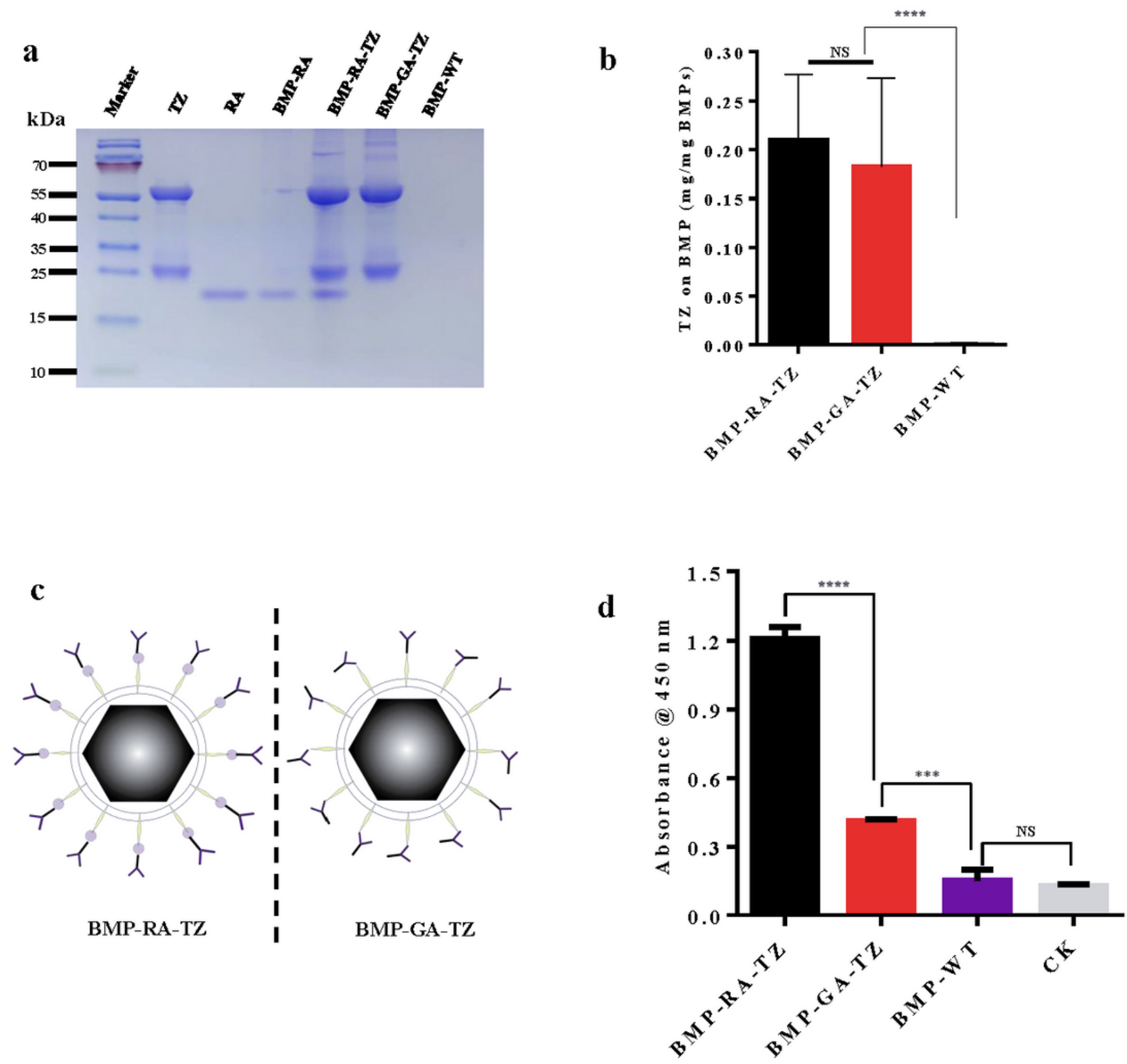

Figure 2 
Incubation of BMP-RAs with TZs led to immobilization of large amounts of TZs on the surface, and formation of BMP-RA-TZ complex. SDS-PAGE and protein assay kit were used to measure TZ amount in the complex. TZ directly linked to BMP-WT by glutaraldehyde (BMP-GA-TZ) was used as control. The engineered BMPs contained similar TZ amounts: $0.2085 \pm 0.04861 \mathrm{mg}$ per mg BMP-RA-TZ and $0.1827 \pm 0.0522$ mg per mg BMP-GA-TZ (Fig. 2a,b).

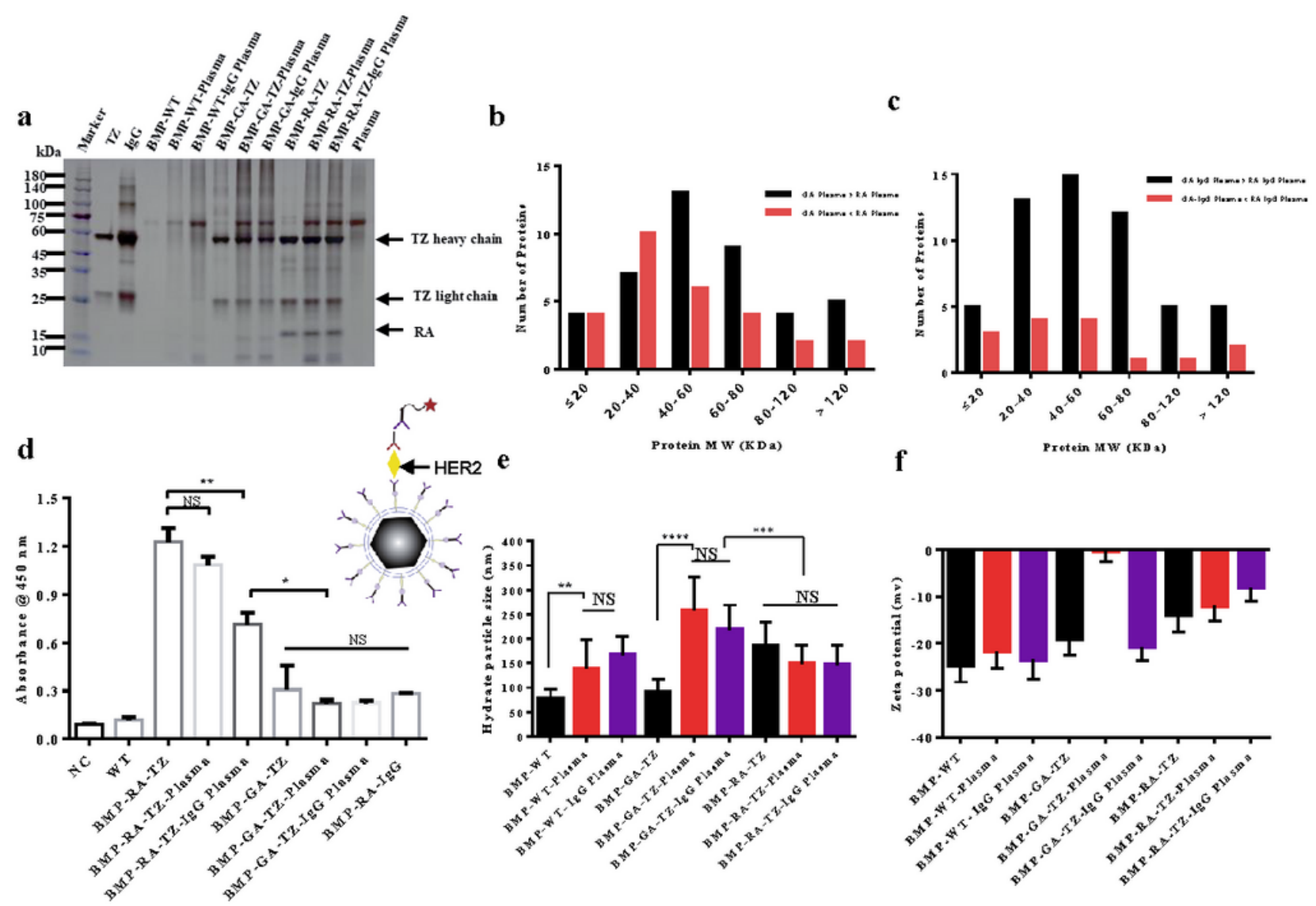

Figure 3

Corona components of engineered BMPs were greatly altered. BMP-RA and BMP-GA, incubated with either normal plasma or IgG plasma, bound much more IgG in the corona than did BMP-WT (Fig. S2). IgG content of BMP-RA-TZ and BMP-GA-TZ coronas was similar to original TZ content (Fig. S3). Components of these BMP coronas were analyzed by liquid chromatography - mass spectrometry (LC-MS). For each type of corona, the 20 proteins with highest content are listed in Table S1. Protein species, amounts, and ratios in coronas were strongly affected by pre-adsorption of TZ and by type of TZ/ BMP linkage (Figs. S3, S4). IgG content in coronas was much higher for BMP-RA-TZ and BMP-GA-TZ than for BMP-WT. IgG content was slightly higher for BMP-RA-TZ-IgG plasma than for BMP-RA-TZ-plasma (Fig. S1). Protein content was higher for BMP-GA-TZ-plasma than for BMP-RA-TZ-plasma. 
a
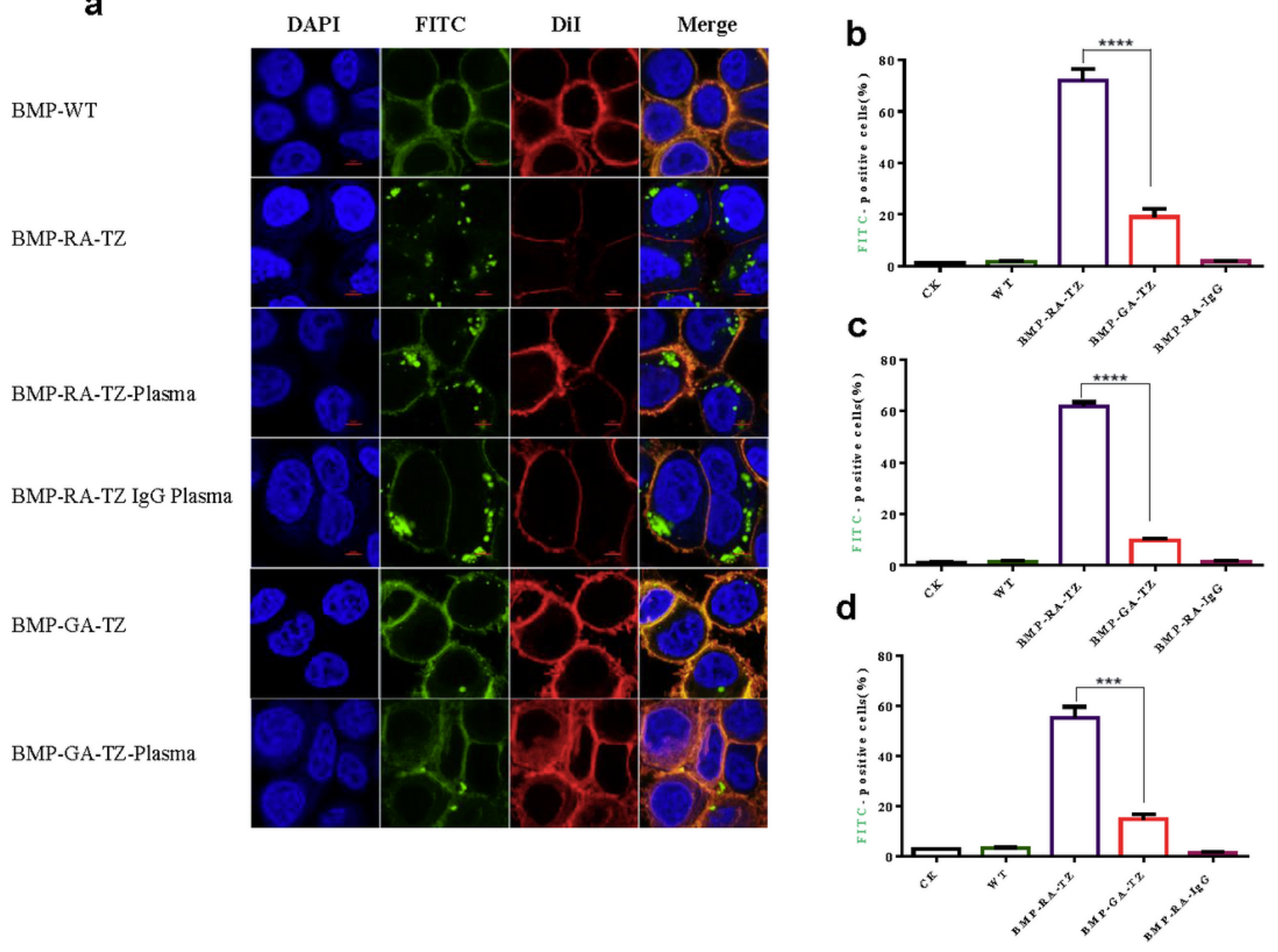

Figure 4

In contrast, BMPs with RA (BMP-RA-TZ, BMP-RA-TZ-plasma, BMP-RA-TZ-IgG plasma) were internalized into cancer cells to some degree, in addition to surrounding the cells (Fig. 4a). 


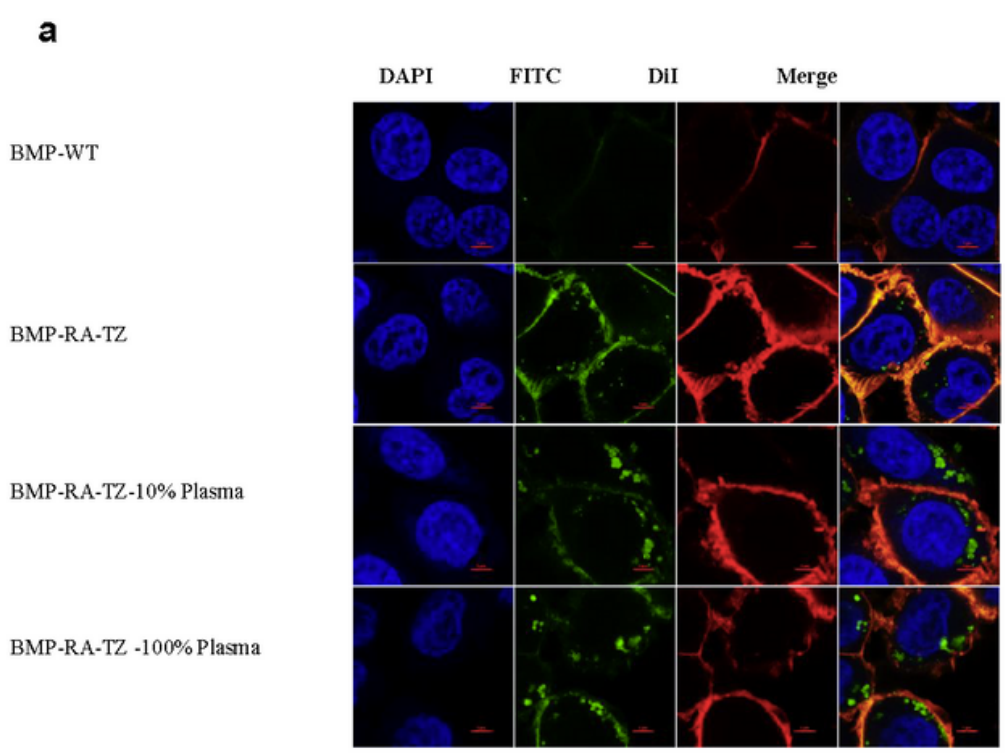

b

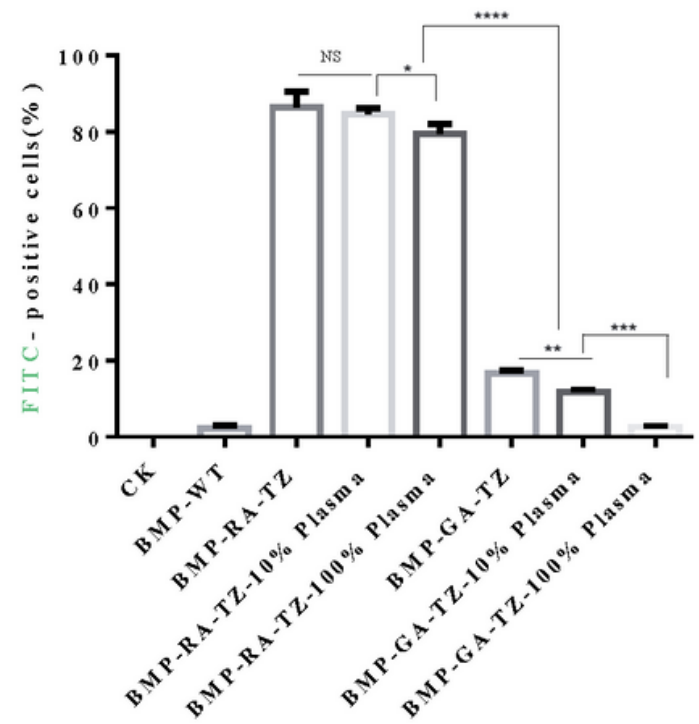

Figure 5

To simulate human body tumor environment, we incubated BMPs, plasma (100\%), and cancer cells together, with staining procedures as described in the preceding section. Treatments with $0 \%$ and $10 \%$ plasma were performed to evaluate effects of plasma concentration on BMP uptake. CLSM observation showed that BMP-RA-TZ was internalized into SK-BR-3 at each of these three plasma levels, whereas BMP-WT was not (Fig. 5a). Flow cytometric analysis revealed FITC-positive percentages in $0 \%, 10 \%$, and $100 \%$ plasma of (respectively) $86.5 \%, 84.6 \%$ and $79.4 \%$ for BMP-RA-TZ, and $16.4 \%, 11.7 \%$ and $2.61 \%$ for BMP-GA-TZ (Fig. 5b). These findings indicate that oriented TZ was required for efficient internalization of BMPs into cancer cells, and that plasma concentration had negligible affect on BMP uptake.

\section{Supplementary Files}

This is a list of supplementary files associated with this preprint. Click to download.

- Table.docx

- SupplementaryInformation.docx

- graphicalabstract.pdf 